\title{
Article \\ What Can We Learn about Science Teachers' Technology Use during the COVID-19 Pandemic?
}

\author{
Liina Adov (D) and Mario Mäeots *(D) \\ Institute of Education, University of Tartu, 50090 Tartu, Estonia; liina.adov@ut.ee \\ * Correspondence: mario.maeots@ut.ee
}

Citation: Adov, L.; Mäeots, M.What Can We Learn about Science Teachers Technology Use during the COVID-19 Pandemic? Educ. Sci. 2021, 11, 255.

https://doi.org/10.3390/

educsci11060255

Academic Editor: Peter Williams

Received: 31 March 2021

Accepted: 18 May 2021

Published: 24 May 2021

Publisher's Note: MDPI stays neutra with regard to jurisdictional claims in published maps and institutional affiliations.

Copyright: (C) 2021 by the authors Licensee MDPI, Basel, Switzerland. This article is an open access article distributed under the terms and conditions of the Creative Commons Attribution (CC BY) license (https:/ / creativecommons.org/licenses/by/ $4.0 /$ )

\begin{abstract}
The purpose of this qualitative research was to describe teachers' experiences in a technologymediated teaching context during the COVID-19 pandemic. We mainly focused on teachers' experiences with technology use (change and variety of the use) and their willingness to use technology in teaching. We designed an interview-based study. The participants were Estonian science teachers who voluntarily agreed to share their experiences about teaching in new and-for most of them - unexpected, distanced learning conditions. Based on teachers' reflections on technology use we could distinguish between three groups in which teachers described different levels of willingness to use technology, change in technology use from pre-COVID to distanced learning, and variety in the use of technology. Our results revealed that the higher teachers' perceived willingness to use technology, the easier it was for them to overcome potential obstacles and cope with the unexpected distanced learning. The main obstacles there were grouped as external (e.g., issues with internet connection, lack of students' digital skills) and internal (e.g., teachers' beliefs about technology use for teaching). I was observed that some obstacles were shared by all teachers (such as limitations on students' digital skills) whereas others where more prevalent in separate groups. This highlights the importance of understanding and considering the variability in the possible obstacles that emerge in using technology in education for teachers with different levels of experience.
\end{abstract}

Keywords: distanced learning; COVID-19; technology-mediated learning; technology-mediated teaching

\section{Introduction}

The pandemic of spring 2020 required teachers to instantly revise their daily teaching practices by endeavouring to apply technological novelty to achieve pedagogical aims. This unexpected pedagogical transformation was smoother for some teachers than others. The COVID-19 pandemic pushed educational systems to jump into the conditions of technology-mediated teaching and learning. This substantial change provided us with an extraordinary opportunity to learn about the role of technology use in teaching in a situation where teachers did not have any other option. Over several decades, researchers have shown that teachers' attitudes predict their willingness to use technology, which in turn predicts how likely they are to engage in the activity [1,2]. Due to this, it has been suggested that the rejection of technology use usually results from low willingness and a negative attitude to using technology. Little is known about what happens when teachers with different levels of willingness are expected to teach using mostly technology. Spoel and colleagues [3] showed that teachers who used technology in their teaching moderately before COVID-19 had a more positive experience in the first month of distanced teaching. This suggests that technology-mediated teaching experiences (e.g., perceived obstacles) may vary based on teachers' previous experiences and willingness to engage with technology. Understanding these experiences gives us the opportunity to acquire a deeper knowledge of which aspects of attitudes and experience should be addressed if we aim to support teachers' purposeful technology use at different levels of willingness and experience after the pandemic as well. 


\subsection{Willingness to Use Technology}

Since the inception of the Theory of Planned Behaviour [4] in the 1980s, behavioural intention has been viewed as the "gate" between attitudes and behaviour, where attitudes can only influence behaviour through changing intention. Behavioural intention has been conceptualized as the willingness to engage in a certain behaviour, or, in Ajzen's words: "A person's readiness to perform a behaviour. This readiness to act can be operationalized by asking whether people intend to engage in the behaviour, expect to engage in the behaviour, are planning to engage in the behaviour, will try to engage in the behaviour, and indeed, whether they are willing to engage in the behaviour" ([5] p. 1122). Therefore, teachers attitudes towards technology in teaching shape how willing they are to utilize it, which in turn predicts the use itself. Based on the previous theoretical model, numerous studies have looked at willingness as a dependent variable (or BI; e.g., [6-8]), with the presumption that without willingness, there will be no behaviour. However, later studies have suggested that the relationship between attitudes, willingness, and behaviour is more dynamic than proposed by the theories borne out of the Theory of Planned Behaviour (TPB). Scherer et al. [9] found similar results on a meta-analysis showing that technology acceptance attitudes predict technology use directly, but not through willingness. Adov, Pedaste, Leijen and Rannikmäe (submitted) [10] found similar results showing that attitudes may predict behaviour above and beyond the willingness-mediated effect. While Scherer et al. [9] used general technology acceptance attitude to aggregate more specific attitude factors, Adov et al. of current manuscript [10] showed that when looking into more specific attitude factors, we can see different relationships with willingness and behaviour. Whereas some attitudes seem to predict others (social influence and facilitating conditions [2]) or relate directly with reported behaviour (social influence and self-efficacy [10]), the evaluations of the usefulness of technology for teaching seem to interact with willingness in predicting behaviour [10]. Tsybulsky and Levin [11] proposed looking beyond individual attitudes and observing the change in teachers' technology-related worldview, which they conceptualized as a system of beliefs where it is not only important to understand the content of the beliefs but their interactions and different patterns of coexistence. The authors used Wilber's (1995) three-dimensional construct to describe an approach to the digital worldview that comprises objective ("how I relate to digital content"), intersubjective ("how I relate to others through digital means"), and subjective dimensions ("how I see myself represented in the digital world"), suggesting that changes in teachers' digital worldview will have an impact, as will changes in pedagogical practices. In one way or another, previous studies show that attitudes, e.g., willingness to use technology, play a role in understanding the behaviour itself.

\subsection{Frequency and Variety in the Use of Technology}

The majority of studies aiming to evaluate the experience of technology usage measure the frequency of the behaviour through surveys, e.g., [12]. Evaluating frequency offers us an insight into the usage of technology; however, it leaves us short on information on how the technology is being used. Studies have also aimed to evaluate the frequency of different activities (e.g., content creation, communication, or information search) for students and teachers that relate to digital literacy or related literacies [13,14]. However, this has still given very limited insights into the complexity and scope of technology use by teachers. Puentedura [15] proposed an SAMR (substitution, augmentation, modification, and redefinition) framework to evaluate the educational use of technology focusing on how technology is integrated into the teaching-learning process. Cromton and Burke [16] used the SAMR framework in a systematic review to evaluate the level of technology implementation based on the level to which it enhances learning experiences. Based on this study, the authors empirically extended the framework and provided an overview of the usage cases for every level of the framework. The first two levels (substitution and augmentation) are collectively named enhancement; on these levels the focus is on substituting usual tasks with similar ones using technology or adding some minimal function, 
such as slides with embedded videos to illustrate the topic [17]. When in substitution, technology acts as a direct substitute for the tool with no functional change, whereas on the second level, augmentation, the direct substitution is enhanced with some functional improvement [16]. The next two levels (modification and redefinition) are named transformative, as the technology used enables one to redefine learning activities up to the point where technology is necessary, as the activity would not be possible in any other way: e.g., collaboratively solving problems or writing text. In the case of modification, technology enables significant task redesign (such as finding stars in the sky using augmented reality), whereas with redefinition technology enables the creation of a new task which would not be possible without technological solutions (such as students recording experiments and editing them to illustrate the learning process) [15]. Cromton and Burke [15] concluded that $54 \%$ of the studies (out of 186) reached the transformational level, while in $46 \%$ of the studies technology was used on the enhancement level. It could be argued that in the case of distanced learning, many technology-mediated activities move towards the transformational level, as it would not be possible to be in contact with students without the mediation of technology. However, the SAMR framework encourages researchers to look one step further and focus on the learning processes and ask if these would be possible without the help of technology.

\subsection{What Makes Technology Difficult to Use?}

Based on research into technology acceptance, teachers' evaluation of how difficult (effort expectancy or perceived ease of use) or useful technology is for teaching is predictive of willingness and ultimately of usage, e.g., [18]. While attempting to better understand the possible obstacles in using technology, researchers have aimed to measure effort expectancy or perceived ease of use, which has been defined as the "degree of ease associated with the use of the system" (p. 450 [18]); in the context of teaching, this can be viewed as the teachers' perceptions of how easy or difficult it would be to use technology for teaching or how inhibiting the foreseen obstacles might be. Bowman et al. [19] divide obstacles to technology use into external barriers which are outside of the teachers' control (e.g., institutional level decisions and infrastructural possibilities) and internal barriers which are under teachers' control (e.g., skills and knowledge, attitudes, and beliefs in technology use). Several studies have suggested that attitudes towards usefulness and effort might overlap to an extent to which their differentiation may not be reasonable [6]. Scherer and colleagues [7] even proposed that obstacles should be viewed as part of usefulness-encountering or anticipating problems with technology use also affects the perceived usefulness of this technology. However, these attitudes have been measured more often than not via questionnaires where teachers can express agreement or disagreement to statements asking them to evaluate whether using technology would be difficult [11]. Yet, this does not give us any information on how teachers see the obstacles that technology poses in teaching and how these might differ for teachers with different willingness and experience in technology use for teaching.

Furthermore, it has been proposed that usefulness might mean different things to teachers based on their goal orientation [20]. For example, a teacher whose goal is to train students for exams will evaluate the usefulness of a technology in teaching differently to a teacher who is aiming to motivate students. Therefore, the perceived difficulties of using technology might depend on the specifics of perceptions-aiming to train students for exams is very likely to create a different set of obstacles in technology use compared with the goal of motivating them. Tondeur and colleagues [21] showed in their meta-synthesis that teachers' beliefs can act as a barrier in technology adoption. Tondeur and colleagues [20] identified three recurrent themes in relation to obstacles in technology use for teachers: lack of time, perceived lack of control (not having an overview of how students are progressing), and traditionalist beliefs (where teachers see no need for technology as traditional practices continue to work). While the first two obstacles may be addressed in technology development by reducing the time it takes to use technological solutions or offering training courses to show teachers that technological solutions take less time than expected, the latter 
requires addressing and changing teachers' beliefs and attitudes. In addition to specific attitudes and beliefs, Tsybulsky and Levin [11] differentiated three groups of teachers based on their digital worldview, reflecting different levels of engagement with the digital world. Even though this was not the main focus of the study, teachers' worldview seemed to be connected to their openness to navigate and explore digital options in teaching. As the authors described, a group of teachers named "outside observers", who reported the lowest level of engagement with all three dimensions of the digital worldview, also expressed in their professional life a lack of interaction, interest, or trust towards the digital solutions. The "circumspect participants" and "conscientious participants", however, reflected more positive attitudes towards the digital world and showed more openness to engage with it. In conclusion, looking into the difficulties expressed by teachers with different levels of willingness and experience with regard to technology use can provide stakeholders with opportunities to address these obstacles.

\subsection{The Present Study}

Due to the COVID-19 pandemic, teachers found themselves in a new relationship with technology and teaching. Interviews give us an opportunity to obtain richer data on the behaviour of the teachers and the possible connections to different aspects of teacher attitudes and experiences.

The aim of the present study is to describe teachers' experiences, e.g., obstacles they encounter, in a technology-mediated teaching context during COVID-19 while taking into account their experiences with technology use (change and variety of the use) and willingness to use technology in teaching.

Therefore, we formulated the following research questions:

1. Which groups of teachers can be distinguished based on the teachers' descriptions of their technology use before and during the COVID-19 pandemic based on three aspects:

willingness to use technology

change in technology use from pre-COVID to distanced learning, and variety in the use of technology?

2. How do the perceived obstacles of technology use differ between groups of teachers?

\section{Methods}

\subsection{Context and Participants}

Our study was conducted in spring 2020 during the COVID-19 pandemic lockdown (as a remark: The Republic of Estonia announced lockdown on the 12 March and the period was officially over on the 17 May). The participants were Estonian science, geography, physics, chemistry, and biology teachers who voluntarily agreed to share their experiences about teaching in new and for most of them unexpected (i.e., unfamiliar teaching practice) distanced learning/teaching conditions. The call to participate was sent to all Estonian basic school STM teachers. In total, there were 13 teachers (see Table 1) from different parts of Estonia who accepted our call to participate in a one-on-one online interview on very short notice during the pandemic.

\subsection{Procedure}

We employed a qualitative study with an interview approach. First, we designed an interview scheme, then tested it with one of the STEM teachers and adjusted the interview questions as needed. The teachers were reached through their school emails inviting them to participate in a study focusing on teacher experience and technology use during the COVID-19 pandemic. One-hour interviews were scheduled and conducted within the lockdown period (April to May 2020); the aim was to collect teachers' experiences in the form of recent and authentic present tense. Participants were informed in advance that the interview would take about an hour. All interviews were conducted via video conferencing systems Zoom or Microsoft Teams and were recorded at least in audio format (except for 
one video in which the recording failed) and as a preventive activity written notes were made during the interview. Oral consent to record the interview was obtained from every participant as the first question with the explanation that the recordings would be used only for achieving the aims of the study and stored on a physical hard drive with access only given to the authors of the current study.

Table 1. Descriptions of participants.

\begin{tabular}{ccc}
\hline Participant (Pseudonym) & Working Experience as a Teacher & Subjects \\
\hline Anna (female) & 3 years & Science and Geography \\
Kati (female) & 2 years & Science and Physics \\
Kristi (female) & 7 years & Science and Chemistry \\
Mati * (male) & 30 years & Physics \\
Karolin (female) & 10 years & Biology and Science \\
Maria (female) & 20 years & Biology \\
Timo (male) & 4 years & Science \\
Kristjan (male) & 64 years & Physics and Science \\
Triinu (female) & 16 years & Biology, Geography, \\
Veiko (male) & 20 years & Science \\
Paul (male) & 17 years & Biology \\
Anne (female) & 34 years & Physics and Chemistry \\
Piret (female) & 35 years & Biology \\
\hline
\end{tabular}

* Note: For this teacher, the interview took place via Microsoft Teams and was not recorded.

\subsection{Instrument}

We collected data with semi-structured interviews (see Appendix A), which provided us with flexibility and possibilities to widen the scope of questions in accordance with the teachers' answers. The main questions of the interview were about how the teachers perceived teaching in distanced learning conditions (e.g., "What are you doing differently in your teaching compared to the period before the distanced learning?") and how they described their use of technology and its variation compared to prior experiences (e.g., "Please describe what role technology plays in your current lessons").

\subsection{Data Analysis}

We used cross-case analysis to describe the similarities and differences between the cases. This enabled us to position these cases relative to each other on three target aspects: willingness to use technology, change in technology use from pre-COVID to distanced learning, and variety in the use of technology. The relevant paragraphs of the interviews were transcribed and analysed using inductive content analysis based on the research questions.

We believe that the replication of the study by others is feasible, as we followed trustworthy principles as described by Williams \& Morrow (2009) [22]:

- Regarding the integrity of the data: interviews were prepared and conducted by the authors of the current paper; authors had a consensual agreement in interpreting data; all interpretations are exemplified with direct quotes from teachers' answers; 4 out of 12 interviews were co-analysed by the authors of the study; any differences in coding were discussed and a common agreement was reached; these interviews were used as referents for the other cases, if needed, to find the relative positioning of the remaining cases; as the interview was in Estonian, all quotes were subsequently translated into English;

- in terms of the balance between reflexivity and subjectivity-in order to check the meaning of the answers given we asked reflective questions during the interview;

- pertaining to the clear communication of findings: all analyses are interpreted and compared with findings from other studies. 


\section{Results}

\subsection{Teachers' Willingness, Change, and Level of Technology Use}

First, we aimed to describe which groups of teachers could be distinguished from one another on the basis of teachers' descriptions of technology use before and during the COVID-19 pandemic based on three aspects: willingness to use technology for teaching, change in technology use (compared to pre-COVID-19), and variety of technology use. In order to give an overview of the participants' relative position on these three aspects, we illustrated the relative positioning of every teacher in our study in Figure 1. Below, we will describe each aspect separately with examples illustrating teachers' relative positioning, which leads us to define the groups of teachers.

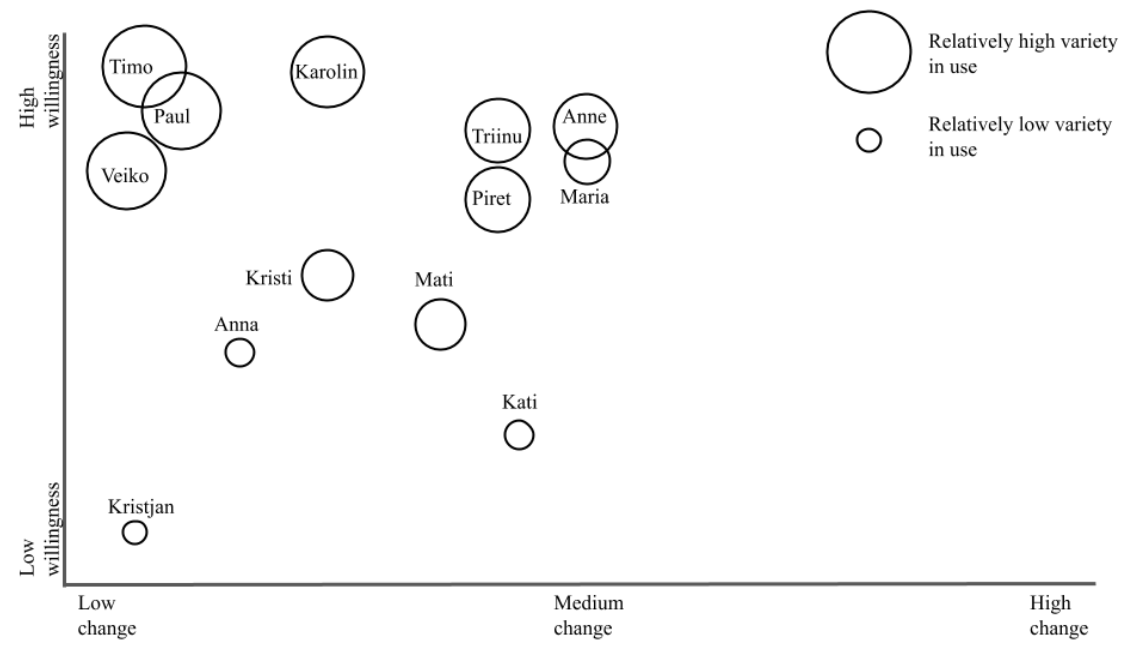

Figure 1. Teachers' relative position on three aspects: willingness to use technology for teaching, change in technology use (compared to pre-COVID-19), and variety of technology use.

\subsubsection{Teachers with High Willingness (Timo, Paul, Karolin, Triinu, Maria, Anne, and Piret)}

We detected a group of teachers who described clearly their willingness to use and try out technological solutions prior to the COVID-19 lockdown who were open to testing different solutions in distanced learning situations.

For example, a teacher with 35 years of experience claimed that she was a frequent technology user and was willing to learn and had already widened her knowledge and skills of using software for conducting video lessons. It is worth noting that she even made comparisons between different kinds of software (e.g., Google Meets, Zoom, Microsoft Teams).

"I was a frequent user already before the crisis and I can say that this has increased during the current situation." (Piret)

They expressed some hesitations on some occasions; however, they saw that these were not something holding them back.

"It was quite a challenge, but not very scary." (Maria)

In many cases, these teachers mentioned that using technology was not an aim in itself and that it would also be important to do things in real life (experiments, etc.), so they did not aim to increase the amount of their technology use. However, they mentioned often their interest in and desire to try out new solutions, as well as their enjoyment at finding a technological solution to their needs and those of their students.

"I am very much pro-technology; I enjoy it when a solution works very well. But we also want to do things in real life." (Karolin)

"I'm aware that it takes more time to prepare lessons, but I'm making efforts to keep pushing at my own pace ... technology is motivational and brings excitement to the students." (Anne) 


\subsubsection{Teachers with Low Willingness (Kristjan and Kati)}

From the interviews, we could see that one group of teachers described a rather low willingness to use technological solutions in teaching before the COVID-19 lockdown, a tendency which also continued during the pandemic under the distanced learning conditions. These teachers reflected being content with the low levels of technology use in their practice and/or an uneasiness in adopting technology in teaching.

"Right now, this digital and distanced learning is still unknown/unusual." (Kristjan)

"I still lean towards the principle that in science subjects (students) should get to do more real experiments and that videos are supportive material." (Kati)

\subsubsection{Teachers with Medium Willingness (Anna, Kristi, and Mati)}

In this group, teachers expressed neutrality or different opinions about technology for teaching. They reported, for example, that they had not been against its use but had not found it very easy to implement.

"I have never been hostile (towards technology), but now it was a bit more difficult ..." (Anna)

"I know there are so many interesting things, but I haven't had the time to make them work for me and haven't had that now either." (Kristi)

In this group, teachers pointed out that they would not change their teaching much once the distanced learning period is over. This had nothing to do with their principles, however, but rather with a hesitation as to how well or how smoothly the implementation would go for them.

"I did not get any braver and will not do things differently than I used to (after the distance teaching)." (Anna)

"I look with envy at how already, only a few days later, younger ones (colleagues) are already working in Classroom (authors' note: program Classroom) and have made their videos with smartphones." (Mati)

\subsection{Technology Use: Change in Behaviour and the Level of Technology Adoption}

In order to describe teachers' behaviour in the changing situation, we addressed two aspects. The first related to changes in technology use in teaching before and during the COVID-19 pandemic. Secondly, we aimed to describe the variety in teachers' technology use, where we focused on the teachers' descriptions of their main aims of technology use and whether these fall into the categories of enhancement or transformational within the SAMR framework, as described above.

Change in Technology Use

None of the teachers reported an extreme leap in their technology use; however, all teachers pointed out that their amount of technology use had risen. For example, one teacher said the following:

"The use of technology (in the classroom) was rising (before the lockdown);

however, now it is absolute." (Timo)

- Low change (Timo, Paul, Karolin, Kristjan, Anna, and Kristi)

From the interviews emerged a group of teachers who described the change in their technology use as minimal. As one teacher said:

"A teacher in a state of emergency is still a teacher." (Timo)

Overall, the teachers in this group did not report much change in the environments they used. For some, this was connected to the fact that they had already used a variety of technological solutions prior to the COVID-19 pandemic. 
"There is not much difference when it comes to the environments that I use." (Anna)

"I haven't had the need to work in anything new (referring to the environments used for teaching), besides Zoom." (Kristi)

"I use about 18 different technological solutions." (Karolin)

In this group, the teachers described change with a focus on their own behaviour with the role of technology in the background, and so even though they clearly described a change, technology use was not central. For example, methods that had previously been used in the classroom were altered to produce technology-mediated versions, e.g., answering or talking to students.

"I give more individual feedback on independent work. Before, I didn't give feedback on every step." (Kristi)

"With some sentences in the e-school, I give instructions on which pages to go through, to which questions to pay extra attention.... Otherwise in the classroom there was oral answering, reporting/answering in front of the class." (Kristjan)

For some teachers, the subtle change was within the focus of how and why they used technology.

"Now, the focus is more on which tools we can use to work together or what they can use for that..." (Timo)

"Before, I checked (Messenger or emails) once a day ... and I did not ... answer right away ... now, when I read it, I answer right away." (Anna)

One teacher emphasized his previous experiences in conducting e-learning, which placed him in good stead. Indeed, previous experience provides an opportunity for a smoother transformation in teaching, but on the other hand, the success of the transformation depends on how flexible the prior material is for modification to new teaching and learning conditions.

"For me, it was nothing new. I have experience of online teaching, I have prepared e-learning materials and conducted video lessons." (Veiko)

- $\quad$ Medium change (Kati, Mati, Piret, Triinu, Anne, and Maria)

Teachers whose stories reflected a medium change described a more substantial change in which the focus was on technology. As one teacher said,

"Everything had to be redone/adapted to a digital version." (Maria)

In some cases, they added that they had used some technological solutions before as well; however, they had preferred not to. During the COVID-19 pandemic, technology became more prevalent than it would have otherwise. As one teacher described,

"For me it has changed substantially. ... Some things we watched before, simulations, videos which connected to physics. But the thought that we have some real things to deal with (authors' note: in the classroom, referring to experiments and discussions in the classroom)." (Kati)

The teachers also noted that the distanced learning period gave them an opportunity to try out things that they had thought about before, or to work on new materials for this occasion:

"(The distanced learning situation) forced me to work on things, to work on things that I was thinking of using, but ... " (Maria)

The teachers also said that even though they had some things "worked in" at the time of the interviews some weeks into the distanced learning period, it takes time.

"Now we have agreed upon which environments we use. Learning new things takes time." (Mati) 
Additionally, teacher's described the maintaining of students' motivation as a reason behind their decision use more technology than before the COVID-19 pandemic.

"For me, technology carries the motivational aim ... students do not like to just fill in worksheets." (Anne)

3.3. The Level of Technology Adoption during the COVID-19 Pandemic (How Teachers Described the Aims of Using Technology)

3.3.1. Enhancement-Focused Adoption (Substitution and Augmentation) (Anna, Kati, Maria, Kristjan, and Kristi)

For one group of teachers, we saw relatively low levels of variety in technology use. They mentioned several environments that they used; we could identify three main categories of aims emerging from the descriptions.

- Instructing

All teachers in this group reported using technology for giving instructions, checking if and how assignments were being done, and giving feedback. They described different environments that helped to fulfil this goal, from pictures of workbooks to files shared via email. Change in this aspect varied among teachers. For some, the tasks were the same, only the sharing was different, for others, the format for the tests changed as well. Two teachers described their somewhat different experiences:

“They send it (referring to a photo of a textbook page) to my email ... and I send it back with my evaluation or comment." (Kristjan)

"I started using Socrative ... now instead of the (open-ended questions) test we do with a multiple-choice test. ... Most of the time goes to giving feedback." (Maria)

In some cases, teachers described how technological solutions set some limitations on giving instructions and controlling processes, indicating that during their usual teaching practice they would have performed their task differently. As one teacher indicated,

"Testing is done more in the multiple-choice test format.... We have less of these longer exams where they had to verbalize (their answers)." (Kristi)

- Communicating

Another aim was communication with students. One teacher explained that previously, they had almost never used emails to communicate with students. Some teachers mentioned the growing role of social media in communication, as it enables seamless, fast communication, and that students would not use other technological solutions as often. As one teacher described,

"Children do not read emails or pick up their phones... Now I am accessible

all the time. ... I need to be on social media all the time." (Maria)

- Sharing materials

Teachers also expressed the need to share materials. The level of and ways in which technology was used for this goal varied between the teachers in these groups as well. For example, one teacher explained how he asked students to use physical materials and share the photos:

"They take a photo of page 14 (referring to the textbook) for which they have read the theory from the book." (Kristjan)

Others described using electronic materials which were either in a written format, such as articles and textbooks, or in a video format. The teachers also shared either existing video materials, videos made by themselves, or used Zoom classes. For example, one teacher reported using,

"e-õpik (collection of electronic textbooks and workbooks), where I ask students

to read the textbook and fill in the workbook" (Anna) 
whereas another noted that,

"To explain the topic further, there are some video clips or a link to an e-textbook." (Kati)

\subsubsection{Transformation-Focused Adoption}

The teachers indicated by a bigger circle in Figure 1 referred to using regularly technological solutions in their teaching, moreover, they referred to this use as an opportunity to change the process of learning. In their descriptions, teachers described using solutions which brought about new possibilities which would not have been accessible for learning with the help of books or workbooks. As one teacher described in the context of giving feedback or checking students' work,

"With the worksheets in Wiser, they can check themselves if they are correct. With the workbooks this doesn't happen, maybe they wait until the teacher collects (the workbooks) ... and then get to know (the right answers), but now they get (them) right away." (Karolin)

Another example of this comes in the form technological co-creation, which became especially valuable during the COVID-19 pandemic.

"Google slides where you can work on something together, rearrange pictures." (Timo)

Interpreting the outcomes described above (willingness to use technology, change in technology use from pre-COVID to distanced learning, and the level of technology adoption) allowed us to distinguish between these three groups of teachers, which helped us to address the second research question. We formed the following groups:

Group A: high willingness, low change, and relatively high variety in use (Timo, Karolin, Paul, and Veiko)

Group B: high willingness, medium change, and average variety (Triinu, Anne, Maria, and Piret)

Group C: low willingness, low to medium change, and low variety (Kristjan, Kati, Anna, Mati, and Kristi).

\subsection{Perceived Obstacles to Technology Use for the Three Groups of Teachers}

After extracting the three groups of teachers based on their experiences, we aimed to describe the differences in their perceived obstacles to technology use (see Table 2). A set of obstacles emerged from each group of teachers, with each group expressing some aspects of the obstacles that were distinct from the others. First, we will cover the overlaps that emerged from the teacher descriptions from the three groups. From there, we will move to the distinctive obstacles for each group.

Table 2. Perceived obstacles by group.

\begin{tabular}{|c|c|c|c|}
\hline Groups & Shared & & Distinctive Obstacles \\
\hline $\begin{array}{c}\text { Group A: Timo, Karolin, Paul, } \\
\text { and Veiko }\end{array}$ & \multirow{3}{*}{$\begin{array}{l}\text { Problems with infrastructure } \\
\text { Problems with giving } \\
\text { immediate feedback }\end{array}$} & - & $\begin{array}{c}\text { Limitations of } \\
\text { technological solutions } \\
\text { Too many solutions in use } \\
\text { in parallel }\end{array}$ \\
\hline $\begin{array}{c}\text { Group B: Triinu, Anne, Maria, } \\
\text { and Piret }\end{array}$ & & \multirow{2}{*}{$\begin{array}{c}\text { Time-consuming for } \\
\text { the teacher } \\
\text { Students' study skills }\end{array}$} & $\begin{array}{l}\text { Difficulties with external } \\
\text { learning materials }\end{array}$ \\
\hline $\begin{array}{c}\text { Group C: Kristjan, Kati, Anna, } \\
\text { Mati, and Kristi }\end{array}$ & & & $\begin{array}{c}\text { Difficulties with } \\
\text { technological solutions } \\
\text { Teachers' attitudes } \\
\text { and beliefs }\end{array}$ \\
\hline
\end{tabular}




\subsubsection{Overlaps Common for all Three Groups}

\section{- Infrastructure}

Teachers pointed out students' lack of access to technology as a possible obstacle. Here, the teachers' environment began to play a role, as according to some teachers, all students had the required technological tools (provided by the school), whereas others reported that regardless of the expectation that everyone has access to tools, they might not.

"All students do not have the means. It is assumed that they would, but they still do not." (Karolin)

Other teachers added that a lack of computers or internet connection can be a problem. However, it was mentioned several times that a solution was usually found; for example, the school administered computers to those students who needed them. Some examples of teachers' experiences include:

"Some have poor internet.... Some have only one computer for the whole family ... there are still a few with these problems." (Maria)

"We heard about some (families) that were lent a computer." (Anna)

- $\quad$ Feedback

Teachers pointed out the lack of immediate feedback as difficulty in teaching solely via technological solutions. This leaves the teacher in the dark as to how much the student understands or if they require any additional support. As one teacher described,

"It is more of a mystical and dark territory (how the student reacts and if they are listening), which makes it also more difficult to adapt the tasks to the specific needs of the student in that situation." (Timo)

Another teacher added:

"I see that everything is filled out (referring to students' work); however, I don't see the state (of the student) behind." (Karolin)

- Students' digital skills

Teachers in all three groups mentioned students' digital skills as one obstacle. Teachers in group A (high willingness, low change, and relatively high variety in use) reflected more resources to overcome these obstacles (previous experience). Here, teachers said that it was important to consider students' skills and choose an environment based on this. One teacher explained:

"I have chosen environments where I know that they have acquired (the skills) already." (Timo)

Another teacher added:

"There are (students) who will never start wanting (to try to figure out environments) and those who can't." (Karolin)

In the other groups, teachers reported that hinderances to do with the lack of student skills also bring with them more general concerns as regards to predicting which environments the students would be able to use on their own. In the teachers' own words:

"Give them a file and they can't use the speller. They know certain things, things that you wouldn't even think of." (Maria)

"There are so many environments, to consider when thinking about which one the student can manage (on her own) at home."

(Anna)

For students' digital skills, we did see a small division between group A and the others, where group A described their previous experience of using digital solutions to help students overcome obstacle, but not for all students. In the other groups, the 
lower previous experience of teachers brought about some inhibiting surprises and more experimenting with different solutions, which added to the workload (new for both the teachers and students).

\subsubsection{Overlap between Group B and Group C}

There were a few obstacles that were not mentioned in group $\mathrm{A}$ but that were described as important obstacles within the two other groups.

- Time-consuming for the teacher

The teachers viewed learning how to use different solutions as being rather timeconsuming, especially in relation to videos, which can be a useful material for the students but take a lot of time for the teacher, thus setting some limits on how accessible this solution is.

"It takes time to learn how to use (different solutions), after that it's useful." (Mati)

"Making videos is extremely time-consuming, I cannot make a video for them for every class." (Maria)

Teachers also described how doing some tasks via technological solutions became more laborious than in the real-life setting - compared to browsing over the student assignments in the classroom, it takes more time digitally as the teacher feels the need to go over every individual assignment.

"Revising is time-consuming, there are no easy, simple solutions for revising (referring to the lack of technological solutions)." (Mati)

Time was also an obstacle in cases where the teacher saw that in the usual teaching situation reliance on a colleague made the preparation time was less costly.

"I'm ready for the technology, but it takes more time for me to prepare materials ... at home, I don't have colleagues who can help ... before it was easier." (Anne)

- Students' study skills

Teachers in this group referred to obstacles beyond technological difficulties, pointing out that for students, working independently was difficult and took more time. Therefore, teachers proposed fewer materials or directed them in a more focused way. Based on the variability within the teachers' use of technology, this obstacle was described on different levels. It was the only obstacle for the following teachers:

"The biggest difficulty is that (students) lack the skills to work on their own with the textbook." (Kristjan)

"More self-discipline is needed, how do I focus on this, how long it takes ... " (Maria)

For other teachers, this was reflected more in the choice of materials shared and the number of assignments given. As one teacher illustrated,

"I have to give things in smaller chunks. In the classroom, I would go through everything in one lesson, now it takes two lessons.... Plus, we don't leave homework, as they do everything at home." (Kati)

\subsubsection{Distinctive Obstacles for the Three Groups}

\section{Group A (Timo, Karolin, Paul, and Veiko)}

In this group, teachers mentioned more distinctly that most of the obstacles they faced were as a result of the distanced learning and were not so clearly connected to technology use for teaching. As one teacher explained,

“The problem is not technology but distance. We do not know and can't evaluate what the situation is (for the student), if they have a place to focus ... " (Timo) 


\section{- Limitations of technological solutions}

Teachers in this group are described as having higher expectations for technology, which in turn overlaps with some obstacles, one of which being that finding environments to fit the needs of their students requires resources which the teacher might lack. One important aspect here was that the teachers perceived the solutions not as too complex (as described by others), but rather not complex enough. This showed that the teachers in this group had very specific pedagogical goals in mind which were not met by the options available to them at the time. This was described by one teacher as follows:

"If I don't have the energy to look that much to pay to find the best solutions for my needs. ... There are not many environments or I don't know how to find them. I want more complex environments." (Karolin)

- Too many solutions in use in parallel

One teacher was teaching in three schools and explicitly stressed that in some cases it was challenging for him to keep track of which technological tool he had to use with the students, as different schools were using different options, e.g., for learning management systems.

"Now all schools are mixed up for me. Earlier I was quite precise with school days (e.g., on Wednesday I'm at one school and on Friday I'm at another school) and I had a clear system. This made it easy to keep track, but now I'm not moving physically from one school to another. Now students contact me at a random time, so I always have to first understand which school, which platform." (Veiko)

\section{Group B (Triinu, Anne, Maria, and Piret)}

Teachers in this group described obstacles through situations where they used additional materials provided by national institutions (e.g., quizzes provided by environmental institutions). They mentioned that in many cases it was not easy to get an overview of the students' results or that the registration process was time-consuming.

"I asked students to take part in a forest quiz ... basically, it meant that I had to manually insert students name by name ... at some point, I thought I would stop, but as I had already added the task description in the e-school, I did finish adding the names of more than 100 students ... it took time." (Triinu)

\section{Group C (Kristjan, Kati, Anna, Mati, and Kristi)}

In this group, the distinctive difficulties were described with a focus on the teachers' perspectives, revealing several topics; teachers noted their own experience as an obstacle alongside student experience.

- Difficulties with technological solutions

Teachers described technological difficulties which limited their options in using the necessary solutions. One teacher described a problem with internet speed/capacity:

"I live in an apartment building, and the internet can just stop working at one point during the day ... there are five class sets of students on the call." (Kati)

Next to student skills, teachers in this group also mentioned their own learning curve alongside obstacles that emerged from the students' low levels of digital skills.

"They (referring to students) cannot use it very well. First of all, you need to teach how, where and then you learn yourself alongside it." (Kati)

Several teachers mentioned that parents had also suggested that students should not spend too much at the computer. On the other hand, teachers highlighted the downsides related to the time spent at the screen by the teachers. One teacher described this from both angles:

"One parent was even upset that so much work is on the computer.... The students may sit there for $30 \mathrm{~min}$, but when checking (student work), the teacher is there longer ... this is tiring for the eyes." (Kati) 
- $\quad$ Teachers' attitudes and beliefs

One of the more elusive obstacles we observed in this group was the teachers' attitudes towards the potential value of digital solutions. Even though the teachers themselves did not mention these explicitly as an obstacle, based on their descriptions of their experiences and principles guiding them in making choices and finding solutions, we observed a clear theme emerging. Attitudes emerged including that non-digital solutions were viewed as preferable or as there was some harm perceived in using more digital solutions.

"I have thought (about using Zoom) but the system that I use ... I am happy and students are happy ... they are used to sending me the worksheets (authors' comment: pictures of worksheets on paper) on time." (Kristjan)

We saw different reasons for this group not engaging with technology use for teaching. In one case, teachers described fundamental principles that led them to limit technology use in teaching. As one teacher describes,

"I took the position (before COVID-19) that as technology is used in many subjects anyway, then in science classes we do 'real things'." (Kati)

\section{Discussion}

The COVID-19 pandemic pushed many educational systems to jump almost entirely into conditions of technology-mediated teaching and learning. This substantial change gave us an extraordinary opportunity to learn about the role of technology use in teaching when teachers did not have any other option. The present study aimed to describe teachers' experiences, e.g., obstacles they encounter, in a technology-mediated teaching context during the COVID-19 pandemic, while taking into account teachers' experiences with technology use (change and variety of the use) and their willingness to use technology in teaching. We addressed this aim in two steps. First, we aimed to group teachers based on their descriptions of technology use before and during the COVID-19 pandemic, considering three aspects: willingness to use technology, change in technology use from pre-COVID to distanced learning, and variety in the use of technology. Secondly, we set out to compare the perceived obstacles of technology use between groups of teachers.

Research question 1: Which groups of teachers can be distinguished based on their descriptions of technology use before and during the COVID-19 pandemic?

The level of willingness distinctly characterized teachers' openness and curiosity to use technology in their teaching before and during the COVID-19 pandemic. Based on these descriptions we saw three levels emerging: teachers with high willingness described their willingness to learn, and their descriptions were more driven by the possibilities that technology provides; in contrast to teachers with medium willingness, who expressed more hesitation; and teachers with low willingness, who expressed some reluctance to using technology in teaching. It can be said that the qualitative descriptions of teachers willingness reflected the previously suggested dynamic relationship between willingness and other attitudes [7,8]. Like Scherer and colleagues [9], we observed a close connection or overlap of several attitudinal aspects, as teachers described the potential usefulness and difficulties of technology use for teaching, to name a few.

From here we looked at the change in technology use, where teachers described a relatively low or medium change in technology use from pre-COVID-19 to teaching during the COVID-19 pandemic. Interestingly, none of the teachers reported an extreme leap in their technology use. Whereas past research has found that the pressure to use technology (as teachers experienced during the COVID-19 pandemic) has a positive impact on its use [23], the present study confirmed this result with some exceptions. Most teachers indeed described some rise in their technology use and described taking relatively comfortable next steps in adopting technology for teaching. However, for some teachers this change was minimal, or, according to their own description, almost non-existent. For example, among teachers with high willingness two trends emerged in reference to their change of their technology use during the COVID-19 situation: we detected teachers with high willingness 
who described a low change in their technology use and those who reflected a medium change. It is important to note here that some teachers in the high willingness group reported that their technology use did not change much as they had used technological solutions prior to the COVID-19 situation. On the other hand, teachers with relatively low or medium willingness described, in some cases, technology use that stayed on the low frequency side, while some described a bigger leap in their use of technology. In the latter cases, teachers mentioned that they had had the intent to use more technological solutions in their teaching before the pandemic and that the COVID-19 situation had given them the nudge to do so. This group's experience follows quite closely what Shin, Han, and Kim proposed in [23].

Acknowledging that the frequency of technology use in teaching might not provide us with information regarding how it is being used, we delved deeper into teachers' descriptions of their technology use before and during the COVID-19 pandemic. Relying on the SAMR model [15], we observed the emergence of different levels of technology use. Looking at these alongside willingness and change in technology use, we could see that descriptions of technology use for teachers with lower willingness expressed enhancement levels. Similar to the findings of Cromton and Burke [16], teachers described using technological solutions (such as taking pictures of homework) as a substitute for their usual teaching practices (checking students' workbooks in the classroom). Descriptions of teachers with relatively higher willingness, on the other hand, more often reflected the transformational level of this framework. As in the Crompton and Burke [16] study, teachers described solutions where technology enables the creation of a new task which would not be possible without technological solutions: for example, asking students to fill in worksheets where they can check themselves if their answer was correct.

Based on the teachers' descriptions we saw the emergence of three groups: (1) teachers with high willingness, low change, and relatively high variety in their technology use (group A); (2) teachers with high willingness, medium change, and average variety (group B); and (3) teachers with low willingness, low to medium change, and low variety (group C, illustrated in Figure 1).

We would also like to highlight a few higher-level observations:

1. Similar to previous studies on willingness and technology use [6-8], we observed based on teachers' descriptions a rather clear connection between willingness and technology use and integration level. Teachers who reflected higher willingness to use technology also described more technology integration within their teaching (before and after the COVID-19 pandemic) and were more likely to be working on the transformational level with this integration. However, when it came to willingness and change in technology use, this relationship was not as straightforward.

2. It is essential to consider that the lack of change in the usage behaviour from preCOVID-19 to the COVID-19 pandemic might not reflect the extent to which teachers use technology in their work. Teachers with relatively high willingness described frequent technology use already before the COVID-19 pandemic; therefore, the change in their technology use was rather low. On the other hand, several teachers whose willingness was relatively low gave a similar description of their technology use during the COVID-19 pandemic. However, we observed that within the medium willingness group the change in technology use from classroom teaching to teaching during the COVID-19 pandemic was quite diverse. Based on this, it could be argued that the impact the distanced teaching situation on the use of technology [23] may vary across the levels of willingness to use technology. To gain more insight into what may inhibit different groups of teachers from adopting technology in their teaching practices, we focused on the kinds of obstacles emerging from the descriptions given by the teachers in these three groups.

Research question 2: How do the perceived obstacles of technology use differ between groups of teachers? 
It is important to note that several obstacles expressed by teachers were shared by either all three groups or two. In the interest of providing insights into how to support teachers in using technology for teaching, we will provide an overview of the shared obstacles and go into more detail about the obstacles shared only by separate groups.

The label "shared obstacles" included three categories: (a) infrastructure (problems with internet connection), (b) feedback (difficulties in providing immediate feedback), and (c) students' digital skills (and lack of students' digital skills). Poor infrastructure is an external obstacle difficult for the teacher to overcome alone and that is usually solved at school level [18]. Thus, it is crucial for schools and local governments to support teachers and students by providing access to the technology necessary for learning, e.g., laptops for home use, as was mentioned by our teachers. As for the second obstacle (difficulties with providing feedback) this overlapped with one recurrent theme in the study by Tondeur and colleagues' [20] on perceived lack of control. The last obstacle highlighted the issue of students' lack of digital skills. This may be viewed as a surprising outcome, as digital competence (including skills) is embedded in the Estonian national curriculum (since 2014) and therefore students' digital skills should not have been an issue.

Group-based obstacles covered areas that included both external and internal issues that teachers experienced during the COVID-19 pandemic. For example, group A reflected on issues relating to the limitations of the technological solutions; some members of the group pointed out that too many solutions in use were not the best way to manage the learning process. The limitation of technology implies that teachers who are at a transformational level set higher expectations for the technology, and when a teacher cannot realize his or her pedagogical goal, he or she perceives it as an obstacle.

Unlike groups B and C, group A did not consider time as an obstacle, or more interestingly, did not mention students' poor learning skills. This finding may be explained by the idea that the teachers in group A had a more extensive collection of learning resources and prior experience of using them. Preparing new or modifying existing learning resources takes time, which is an inhibiting factor for teachers when it comes to integrating technology into the classroom [24]. In addition, we can see that this group of teachers resembles the "conscientious participants" in the research by Tsybulsky and Levin [11], where teachers described seeing more opportunities and less difficulties in using new technologies. The adoption of this worldview may have meant that some of the obstacles described by the other groups of teachers, such as groups B and C in our case, were not perceived as obstacles by teachers with a digital worldview.

Obstacles within group B related to the continuing process of seeking new ways and technologies to support pedagogical goals. However, this was a complicated process for this group, as they claimed that they missed support from colleagues and thus, it took more effort. This was confirmed by their average variety in technology use. Tsybulsky and Levin [11] described similar experiences of teachers who indicated their desire to be more able to use technological solutions to be there for their students, but saw some difficulties engaging with the technology themselves.

The last group of teachers, group C, indicated difficulties that related to technological solutions (external barriers) and teachers' own attitudes or beliefs (internal barriers) about integrating technology. The latter is consistent with the work of Liu et al. [25], which looks into teacher confidence and comfort using technology in teaching. Although we could expect a change in technology use [23], interestingly, the pressurized situation of the pandemic did not reinforce the teacher disposition to use technology and did not increase their confidence in using technology. As Tsybulsky and Levin [11] noted, changing a set of beliefs or worldview which may help a person's relationship with technology in the distanced leaning environment is a long and gradual process which requiring a comprehensive approach. As seen from the descriptions of group C, having situational pressure is not enough as hesitations about technology and the digital world outweighs any external pressure. 


\section{Conclusions}

Through teachers' reflections on teaching during the COVID-19 pandemic, the current study presented several observations and potential bottlenecks in the field of technology adoption by teachers. In addition, through these reflections we were able to take a deeper look at the reasons behind frequency of technology use. We saw that teachers with higher willingness, situated on a more transformative level of technology use, described external obstacles, while teachers with a lower willingness and working at the enhancement level of technology use reported both internal and external obstacles. These internal obstacles included beliefs towards technology, which may go undetected when asking teachers to report their attitudes via a questionnaire. The identification of these obstacles might help us better understand the hesitation in adopting technology for teaching and exploring the possibilities of transforming the learning experiences through technology. However, it is important to note the understanding that teachers with high willingness have different needs for support and training in technology use compared to teachers with relatively low willingness.

\section{Limitations and Implications for Further Research}

One limitation of this qualitative study is that the sample used was not representative of the whole population of Estonian science teachers; thus, generalizations are not possible. Another, possibly the most important, limitation (this could also be viewed as a strength) is that similar COVID-19 pandemic conditions will be difficult to repeat as the situation will have lost its novelty for teachers. During our study, teachers did not know what to expect, and, moreover, they did not have any time to prepare for changes in their teaching.

As participation in the study was voluntary, it could be argued that teachers with certain openness towards the topic of the study were more prone to participate. Even though we saw that there was variety in the experiences teachers described in their teaching with technology, it is relevant to be mindful of possible participation bias. Future studies could take this into account, aiming to recruit teachers based on random sampling.

In this study, we did not pay extra attention to the characteristics of the schools in which our participants worked. As mentioned by a few teachers, these characteristics might be connected to possible obstacles and possibilities for the teachers to overcome these (e.g., is the school able to help students with computers).

In terms of future research, it would be useful to extend the current findings by examining schools to identify internal obstacles and their role in teaching during distanced learning. Another interesting future perspective would be to have follow-up interviews with the same teachers in order to explore longitudinal views on their teaching experiences throughout the pandemic.

Author Contributions: Conceptualization, L.A. and M.M.; methodology, L.A. and M.M.; validation, L.A. and M.M.; formal analysis, L.A. and M.M.; investigation, L.A. and M.M.; data curation, L.A. and M.M.; writing — original draft preparation, L.A. and M.M.; writing—review and editing, L.A. and M.M.; visualization, L.A. All authors have read and agreed to the published version of the manuscript.

Funding: This work was sponsored by the Estonian Research Council (ERC) through the institutional research funding project "Smart technologies and digital literacy in promoting a change of learning" (Grant Agreement No. IUT34-6).

Institutional Review Board Statement: The study was conducted according to the guidelines of the Declaration of Helsinki.

Informed Consent Statement: Informed consent was obtained from all subjects involved in the study.

Data Availability Statement: Not applicable.

Conflicts of Interest: The authors declare no conflict of interest. 


\section{Appendix A}

Semi-structured interview questions applied in the study:

A. Descriptive questions

1. What does it mean to be a teacher working under distanced learning conditions?

2. What are you doing differently in your teaching compared to the period before the distanced learning?

3. What has been the biggest change in preparing the lessons; in conducting the lessons; and in giving feedback to the students.

\section{B. Technology use in teaching}

1. What role does technology play in your current lessons? How does it differ from before?

2. What kind of technology do you usually apply to your lessons?

3. What are the main goals of using technology in your lessons during distanced learning? Give some examples.

a. What are the main obstacles to using technology under distanced learning conditions?

b. What is positive about using technology under the conditions of distanced learning?

c. Does distanced learning change your attitude to technology?

4. What were the main goals of using technology in your lessons before distanced learning?

Background

1. Age

2. Years taught

3. Subject(s) taught

\section{References}

1. Scherer, R.; Teo, T. Editorial to the special section-Technology acceptance models: What we know and what we (still) do not know. Br. J. Educ. Technol. 2019, 50, 2387-2393. [CrossRef]

2. Adov, L.; Pedaste, M.; Leijen, Ä.; Rannikmäe, M. Does it have to be easy, useful, or do we need something else? STEM teachers' attitudes towards mobile device use in teaching. Technol. Pedagog. Educ. 2020, 29, 511-526. [CrossRef]

3. Van der Spoel, I.; Noroozi, O.; Schuurink, E.; van Ginkel, S. Teachers' online teaching expectations and experiences during the Covid19-pandemic in the Netherlands. Eur. J. Teach. Educ. 2020, 43, 623-638. [CrossRef]

4. Ajzen, I. From Intentions to Actions: A Theory of Planned Behavior. In Action Control: From Cognition to Behavior; Kuhl, J., Beckmann, J., Eds.; Springer: Berlin/Heidelberg, Germany, 1985; pp. 11-39. [CrossRef]

5. Ajzen, I. The theory of planned behaviour: Reactions and reflections. Psychol. Health 2011, 26, 1113-1127. [CrossRef]

6. Kabakçi-Yurdakul, I.; Ursavas, Ö.F.; Becit-Isçitürk, G. An Integrated Approach for Preservice Teachers' Acceptance and Use of Technology: UTAUT-PST Scale. J. Educ. Res. 2014, 21-36. [CrossRef]

7. Scherer, R.; Siddiq, F.; Teo, T. Becoming more specific: Measuring and modeling teachers' perceived usefulness of ICT in the context of teaching and learning. Comput. Educ. 2015, 88, 202-214. [CrossRef]

8. Scherer, R.; Siddiq, F.; Tondeur, J. The technology acceptance model (TAM): A meta-analytic structural equation modeling approach to explaining teachers' adoption of digital technology in education. Comput. Educ. 2019, 128, 13-35. [CrossRef]

9. Scherer, R.; Siddiq, F.; Tondeur, J. All the same or different? Revisiting measures of teachers' technology acceptance. Comput. Educ. 2020, 143, 103656. [CrossRef]

10. Adov, L.; Pedaste, M.; Leijen, Ä.; Rannikmäe, M. Moving from intention to behaviour: Predicting teachers' mobile device use for teaching. (submitted).

11. Tsybulsky, D.; Levin, I. Science teachers' worldviews in the age of the digital revolution: Structural and content analysis. Teach Teach. Educ. 2019, 86, 102921. [CrossRef]

12. Al-Emran, M.; Mezhuyev, V.; Kamaludin, A. Technology Acceptance Model in M-learning context: A systematic review. Comput. Educ. 2018, 125, 389-412. [CrossRef]

13. Kippers, W.B.; Poortman, C.L.; Schildkamp, K.; Visscher, A.J. Data literacy: What do educators learn and struggle with during a data use intervention? Stud. Educ. Eval. 2018, 56, 21-31. [CrossRef]

14. Hatlevik, O.E.; Ottestad, G.; Throndsen, I. Predictors of digital competence in 7th grade: A multilevel analysis. J. Comput. Assist. Learn. 2014, 31, 220-231. [CrossRef]

15. Transformation, Technology, and Education. Available online: http://hippasus.com/resources/tte/ (accessed on 30 March 2021).

16. Crompton, H.; Burke, D. Mobile learning and pedagogical opportunities: A configurative systematic review of PreK-12 research using the SAMR framework. Comput. Educ. 2020, 156, 103945. [CrossRef] 
17. Wahyuni, S.; Mujiyanto, J.; Dwi, R.; Fitriati, S. Teachers' Technology Integration into English Instructions: SAMR Model. In Proceedings of the International Conference on Science, Education and Technology (ISET 2019), Semarang, Central Java, Indonesia, 29 June 2019. [CrossRef]

18. Venkatesh, V.; Morris, M.G.; Davis, G.B.; Davis, F.D. User acceptance of information technology: Toward a unified view. MIS Q 2003, 27, 425-478. [CrossRef]

19. Bowman, M.A.; Vongkulluksn, V.W.; Jiang, Z.; Xie, K. Teachers' exposure to professional development and the quality of their instructional technology use: The mediating role of teachers' value and ability beliefs. J. Res. Technol. Educ. 2020, 1-17. [CrossRef]

20. Karaseva, A.; Pruulmann-Vengerfeldt, P.; Siibak, A. Relationships between in-service teacher achievement motivation and use of educational technology: Case study with Latvian and Estonian teachers. Technol. Pedagog. Inf. 2018, 27, 33-47. [CrossRef]

21. Tondeur, J.; Roblin, N.P.; van Braak, J.; Voogt, J.; Prestridge, S. Preparing beginning teachers for technology integration in education: Ready for take-off? Technol. Pedagog. Educ. 2017, 26, 157-177. [CrossRef]

22. Williams, E.N.; Morrow, S.L. Achieving trustworthiness in qualitative research: A pan-paradigmatic perspective. Psychother. Res. 2009, 19, 576-582. [CrossRef] [PubMed]

23. Shin, W.S.; Han, I.; Kim, I. Teachers' Technology Use and the Change of Their Pedagogical Beliefs in Korean Educational Context. Int. Educ. Stud. 2014, 7, 11-22. [CrossRef]

24. Harrell, S.; Bynum, Y. Factors Affecting Technology Integration in the Classroom. Educ. Manag. Adm. Leadersh. 2018, 5, 12-18.

25. Liu, F.; Ritzhaupt, A.D.; Dawson, K.; Barron, A.E. Explaining technology integration in K-12 classrooms: A multilevel path analysis model. Educ. Technol. Res. Dev. 2017, 65, 795-813. [CrossRef] 